\title{
A novel AAGAB mutation in a Peruvian family with punctate palmoplantar keratoderma
}

\author{
Ana C. Gómez-García ${ }^{1^{*}}$, Julio C. Salas-Alanís ${ }^{2,3^{*}}$, Nathaly Bar-Fernandez ${ }^{1}$, Rossana Mendoza-Meza ${ }^{1}$, Stephane M. Díaz-

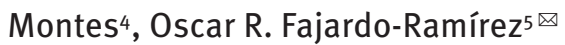

${ }^{1}$ Department of Dermatology, Peru National Police Hospital, Lima, Peru. ${ }^{2} J a l i s c o$ Dermatological Institute, Guadalajara, Mexico. ${ }^{3}$ Secretary of Health

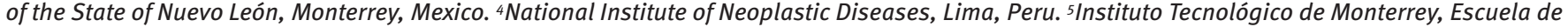
Medicina y Ciencias de la Salud, Monterrey, México.

\begin{abstract}
Buschke-Fischer-Brauer (BFB) disease is a rare keratoderma characterized by multiple hyperkeratotic lesions on the palms and soles, with an autosomal dominant pattern. In several countries, some genetic alterations have been associated with this clinical entity. A 68-year-old Peruvian woman presenting with hyperkeratotic lesions on both her palms and soles was diagnosed with BFB keratoderma. After sequencing of the genes that had previously been related to this disease, a mutation (C.249C $>G$ ) that was predicted to generate a termination codon (Tyr83*) was found in the alpha and gamma adaptin binding protein P34 gene (AAGAB). After treatment with $30 \%$ urea plus $10 \%$ salicylic acid, the patient experienced an improvement in her condition. Here we report a novel mutation in the AAGAB gene of a patient diagnosed with BFB keratoderma and a treatment that improved her symptoms.
\end{abstract}

Keywords: keratoderma, mutation, AAGAB

Received: 7 September 2020 | Returned for modification: 6 January 2021 | Accepted: 5 February 2021

\section{Introduction}

Punctate palmoplantar keratoderma (PPK), also referred as Buschke-Fischer-Brauer (BFB) disease, is a very rare genetic disease characterized by punctate hyperkeratotic papules affecting the palmar and plantar skin (1). Its prevalence is estimated to be 1.17 per 100,000, and the clinical manifestations for this entity are observed between 10 and 45 years of age (2). Although the etiology remains poorly understood, the loci located at 8q24.13-8q24.21 and 15q22-15q24 have been associated with PPK. Thorough analysis has shown that the collagen type XIV alpha 1 chain (COL14A1) and alpha and gamma adaptin binding protein p34 (AAGAB) genes, located in these loci, respectively, present pathogenic mutations in patients diagnosed with PPK and their family members (3). The AAGAB gene codes a 315 amino-acid protein that allows interaction between the gamma-adaptin and alpha-adaptin subunits to stabilize the $\mathrm{AP} 2$ adaptor during clathrin-mediated endocytosis (CME) (4). To date, more than 20 mutations affecting the AAGAB gene have been reported in families from several countries such as Mexico, Japan, Ireland, and Scotland (5). Here we describe a novel mutation in the AAGAB gene in a woman diagnosed with PPK, who referred other members of her family that presented the same clinical manifestations.

\section{Methods}

After clinical examination, informed consent from the patient was obtained and a blood sample was taken in tubes containing EDTA (BD Vacutainer, Franklin Lakes, NJ, USA). DNA was isolated using the DNeasy kit from Qiagen (Hilden, Germany). The exome was sequenced with next-generation sequencing (NGS) in the Illumina NovaSeq platform using the Agilent SureSelect Clinical Research Exome v3. Three hundred paired end-run cycles with a Phred qual- ity threshold $>30$ were executed. The genetic variants detected were aligned against Homo sapiens, hg19, build 37.2, and analyzed using Variant Studio1.o Software (Illumina Inc.). Mutation severity, on the basis of mutation effects in the amino-acid sequences, was estimated using the Sorting Intolerant From Tolerant (SIFT) and Polymorphism Phenotyping (PolyPhen2) algorithms.

\section{Case report and results}

A 68-year-old Peruvian female patient was admitted for valve replacement surgery due to mitral cardiomyopathy; she also attended the department of dermatology due to hyperkeratotic lesions on both her palms and soles (Figs. $1 \mathrm{~A}$ and $1 \mathrm{~B}$ ). The patient stated that the lesions had appeared when she was young, and she received a homemade herbal remedy that was topically applied, with no improvement at all. She also described the lesions as itchy and making it difficult for her to carry out daily activities; these inconveniences disappear when the lesions are surgically removed. She mentioned that her father and one of her brothers presented similar lesions, as well one of her daughters and one nephew (the son of her affected brother; Fig. 1C).

Physical examination revealed multiple hyperkeratotic papules and nodes with central depression and diameters ranging from 4 to $10 \mathrm{~mm}$ on both palms; the same characteristics were present on the soles, mostly observed in pressure areas.

A skin biopsy stained with H\&E showed hyper-granulosis and hyperkeratosis with a well-defined central epidermal depression and coronoid lamella (Fig. 1D). Neither the laboratory tests nor radiographies showed any subjacent pathological alteration.

Following informed consent from the patient, a blood sample was obtained, and the patient's DNA was isolated and sequenced using NGS. Subsequent analysis of the sequestered sequence data indicated the presence of a heterozygote substitution c.249C $>\mathrm{G}$,

*Ana C. Gómez-García and Julio C. Salas-Alanís contributed equally to this article, and both should be considered first author. 
mapped to chromosomal region 15q22-15q24, which led to premature truncation of gene AAGAB by introduction of a new stop codon at amino-acid position $83\left(\right.$ Tyr83 $\left.3^{\star}\right)$, aligned with previous reports of AAGAB mutations causing punctate PPK type 1. Surprisingly, a search in databases such as ClinVar from NCBI did not show any previous report of this truncated version of the AAGAB protein. The patient was treated topically with $30 \%$ urea plus $10 \%$ salicylic acid, and she showed clinical improvement.

\section{Discussion}

As reported by Polher et al., a deficiency of p34 caused by mutations in the AAGAB gene results in hyperproliferative hyperkera-

A

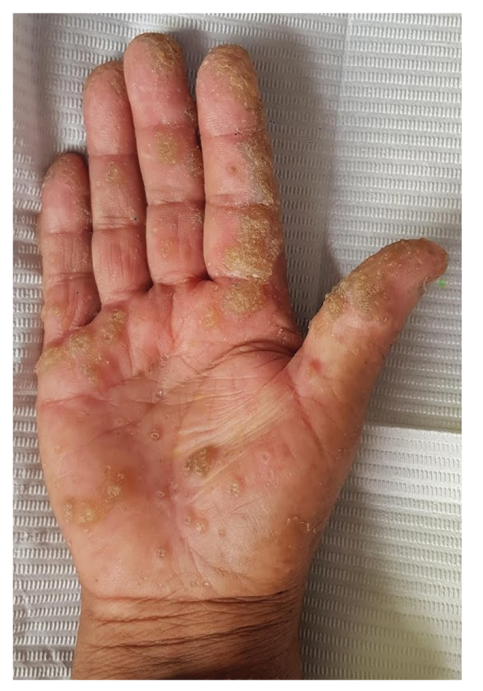

tosis due to constant activation of epidermal growth factor receptor (EGFR) as a result of defective endocytosis and recycling of the EGFR proteins (4). In this context, Giehl et al. were the first to demonstrate a possible relationship between p34 and PPK (6). Here we describe a novel stop codon mutation in the AAGAB gene generating a truncated version of p34 in a Peruvian woman diagnosed with PPK, a rare autosomal dominant disease. In addition, the patient experienced clinical improvement after topical treatment with 30\% urea plus 10\% salicylic acid. Poor access to the city where the patient lives makes it difficult to carry out a full genetic analysis of other members of her family, or to obtain more samples to carry out an extensive analysis to confirm the absence of the protein and the consequences of this.

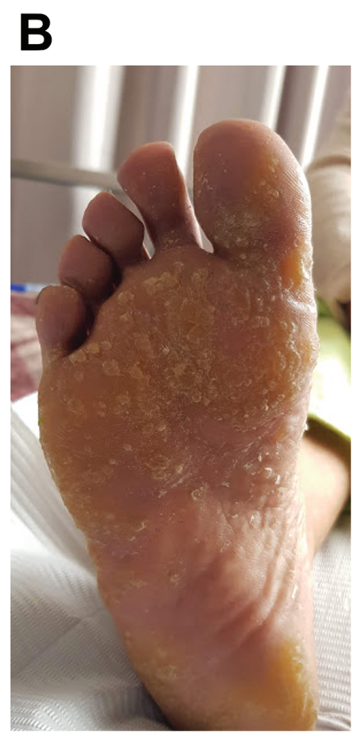

D

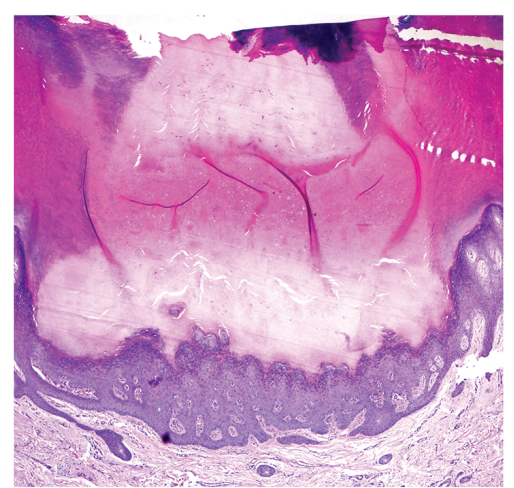

Figure 1 | Clinical data from the patient: characteristic papules on the palms (A) and soles (B) diagnosed as punctate palmoplantar keratoderma (PPK); her family genetic tree (C); and a skin biopsy stained with $\mathrm{H} \& \mathrm{E}$ showing regular acanthotic epidermis and hypergranulosis associated with a prominent stratum corneum (D).

\section{References}

1. Bukhari R, Alhawsawi W, Radin AA, Jan HD, Al Hawsawi K, Al Ahmadi M. Punctate palmoplantar keratoderma: a case report of type 1 (Buschke-Fischer-Brauer disease). Case Rep Dermatol. 2019;11:292-6.

2. Charfeddine C, Ktaifi C, Laroussi N, Hammami H, Jmel H, Landoulsi Z, et al. Clinical and molecular investigation of Buschke-Fischer-Brauer in consanguineous Tunisian families. J Eur Acad Dermatology Venereol. 2016;30:2122-30.

3. Antonio JR, Oliveira GB, Rossi NCP, Pires LGG. Exuberant clinical picture of Buschke-Fischer-Brauer palmoplantar keratoderma in bedridden patient. An Bras Dermatol. 2014;89:819-21.
4. Pohler E, Mamai O, Hirst J, Zamiri M, Horn H, Nomura T, et al. Haploinsufficiency for AAGAB causes clinically heterogeneous forms of punctate palmoplantar keratoderma. Nat Genet. 2012;44:1272-6.

5. Li M, Dai X, Cheng R, Yang L, Yao Z, Liu J. A novel 5-bp deletion mutation in $A A G A B$ gene in a Chinese family with punctate palmoplantar keratoderma. Acta Derm Venereol. 2014;94:339-40.

6. Giehl KA, Eckstein GN, Pasternack SM, Praetzel-Wunder S, Ruzicka T, Lichtner P, et al. Nonsense mutations in AAGAB cause punctate palmoplantar keratoderma type Buschke-Fischer-Brauer. Am J Hum Genet. 2012;91:754-9. 